\title{
Variations intraspécifiques de la taille des ailes et du thorax chez G lossina palpalis palpalis (Robineau-D esvoidy, 1830) en zone forestière de Côte d'Ivoire
}

\author{
B. Sané ${ }^{1}$ P. Solano ${ }^{1}$ A. Garcia ${ }^{2}$ \\ F. Fournet ${ }^{3}$ C. Laveissière ${ }^{4}$
}

Mots-clés

Glossina palpalis palpalis - Aile Thorax - Mesure - Facteur climatique Côte d'Ivoire.

\begin{abstract}
Résumé
La morphométrie du thorax et des ailes a été étudiée sur 497 individus de Glossina palpalis palpalis capturés en lisière d'un village du centre-ouest de la Côte d'Ivoire. Des différences significatives de taille ont été mises en évidence selon le sexe et la saison de capture. Les glossines en fin de saison des pluies avaient une taille plus grande que celles capturées pendant la saison sèche. La relation entre la taille des individus qui composent une population de glossines et les conditions climatiques pourrait avoir des conséquences sur la capacité vectorielle. De récents développements dans les méthodologies d'analyse permettront d'affiner ces études.
\end{abstract}

\section{INTRO DUCTION}

Glossina palpalis palpalis, vecteur majeur des trypanosomoses en zone forestière de Côte d'Ivoire, se rencontre aussi bien dans les zones de plantations que dans les lisières de village (15). Les études réalisées sur la morphométrie des glossines ont montré qu'il était possible de distinguer des communautés de glossines qui colonisent différents biotopes $(8,11,12,13,17)$.

Dans cette étude, la surface moyenne du thorax et la longueur des ailes de G. p. palpalis capturée en lisière de village ont été mesurées, avec pour objectif de tenter de distinguer au cours du temps différentes populations de cette sous-espèce de glossine dans ce biotope. De telles études pourraient en effet permettre d'établir une relation entre la taille des glossines et les conditions du milieu où elles vivent $(4,10,21)$. Cette relation pourrait avoir des conséquences sur la capacité des glossines à transmettre la maladie puisque la taille des individus émergents est corrélée à leurs réserves énergétiques (lipidiques) et au taux de mortalité $(6,21)$.

1. Institut Pierre Richet, Organisation de coordination et de coopération pour la lutte contre les grandes endémies (Occge), BP 1500 Bouaké, Côte d'Ivoire

2. Institut de recherche pour le développement (Ird, ex-Orstom), BP 1386, Dakar, Sénégal

3. Laboratoire Espace, santé et territoire/Isd, 75006 Paris, France

4. Organisation de coordination pour la lutte contre les endémies en Afrique centrale (Oceac), BP 288, Yaoundé, Cameroun

\section{MATERIEL ET METHODES}

Les études ont été menées en lisière du village de Krikoria. Ce village est situé au centre-ouest de la Côte d'Ivoire en zone forestière, dans le foyer de trypanosomose humaine (ou maladie du sommeil) de Zoukougbeu. Il est éloigné des plantations et des îlots forestiers et une jachère l'entoure.

Le piège biconique (7) a été utilisé en capture continue (pendant quatre jours) pour la collecte des glossines présentes en lisière du village. Les captures des glossines ont été réalisées à différentes périodes de l'année : en saison sèche froide (janvier), en saison chaude (juillet) et en saison humide (septembre, octobre). Les sites de captures de la lisière du village ont été les mêmes durant toute l'expérimentation.

La taille des ailes et celle du thorax des deux sexes de G. p. palpalis ont été établies selon la technique de Pollock et coll. (20) (figure 1) en mesurant :

- le tranchant (x) de la cellule en hache (ou cellule discale) de chacune des deux ailes ;

- la distance entre les points d'insertion des plus grandes soies humérales situées de chaque côté du thorax (y) et entre la base des soies scutellaires médianes et la suture transverse antérieure mésonotale (y). La surface du thorax de la glossine a été obtenue par multiplication de ces deux distances. 


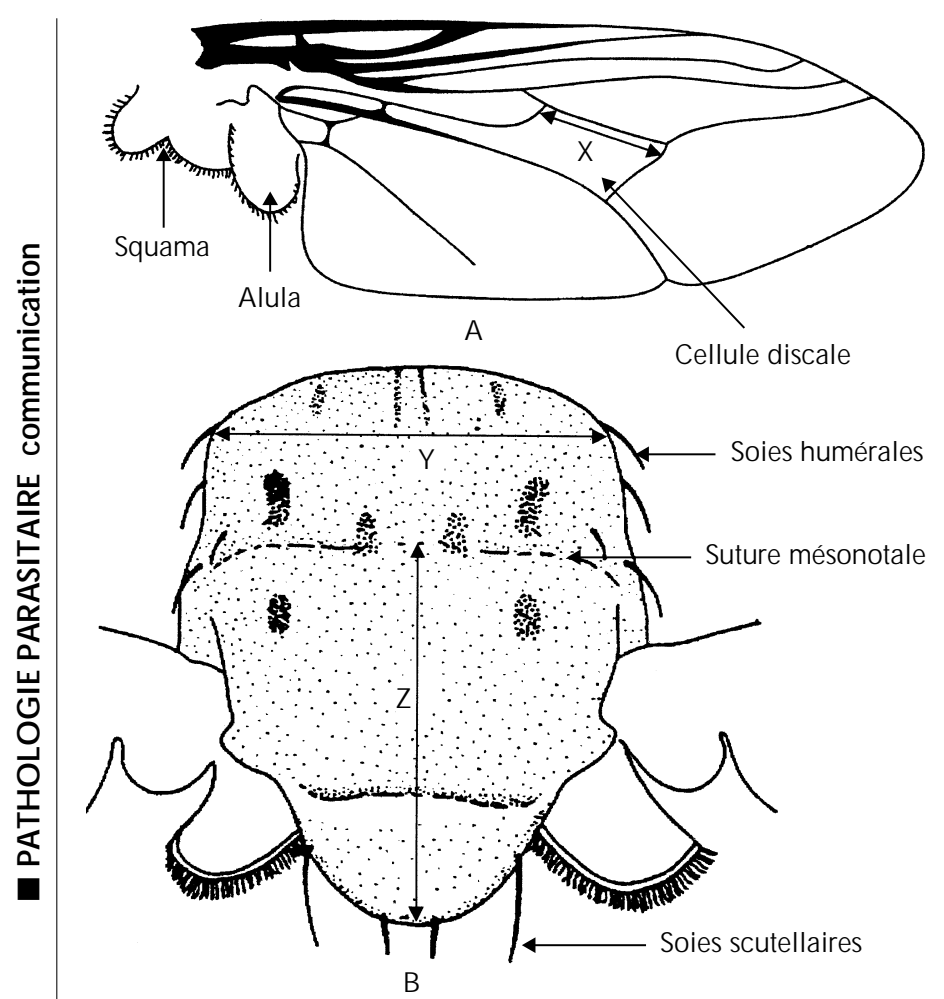

Figure 1 : mensuration de l'aile (A) et du thorax (B) des glossines ; X, y, z sont les longueurs mesurées. Source : J.N. Pollock, 1980, Fao.

Les mesures ont été faites sous une loupe binoculaire possédant un micromètre oculaire étalonné au préalable avec une lame graduée en millimètres. Après vérification de la normalité des données, des tests de comparaison de moyennes ont été effectués (probabilité $\mathrm{P}$ associée au test $\mathrm{T}$ de Student).

\section{RESU LTATS}

Au cours des quatre sessions de piégeage, le thorax et les ailes de 497 (250 femelles et 247 mâles) G. p. palpalis, capturées en lisière de village, ont été mesurés.

\section{Surface du thorax}

Les surfaces moyennes des thorax de $G$. $p$. palpalis mâle et femelle capturées en lisière de village sont présentées à la figure 2 . Les valeurs des surfaces moyennes des thorax des femelles ont été significativement supérieures (de 21 p. 100 en moyenne) à celles des mâles quel que soit le mois considéré $\left(\mathrm{p}<10^{-4}\right)$. Elles ont été maximales en octobre, fin de la saison des pluies, et minimales en juillet, saison chaude qui suit la première saison des pluies, pour les deux sexes.

Chez les mâles comme chez les femelles, au cours des saisons, seule la valeur obtenue en octobre a été significativement supérieure à toutes les autres valeurs (tableau I). Les autres variations intersaisonnières n'ont pas été significatives.

\section{Taille de la cellule en hache}

Les longueurs moyennes des cellules en hache des glossines mâles et femelles capturées en lisière du village de Krikoria sont illustrées à la figure 3. Les femelles avaient une cellule discale significativement plus grande (de 15 p. 100 en moyenne) que les mâles quelle que fût la saison $\left(\mathrm{p}<10^{-4}\right)$.
Les valeurs maximales des mesures ont été atteintes au mois d'octobre chez les deux sexes. Elles ont été minimales en juillet pour les mâles et en janvier pour les femelles.

Au niveau des variations intersaisonnières, les mesures ont varié significativement chez les mâles entre le mois de juillet, où la valeur a été minimale, et toutes les autres périodes de capture. Ces variations ont été moins marquées chez les femelles; seules les valeurs extrêmes ont été significativement différentes (entre les mois de janvier et d'octobre) (tableau II).

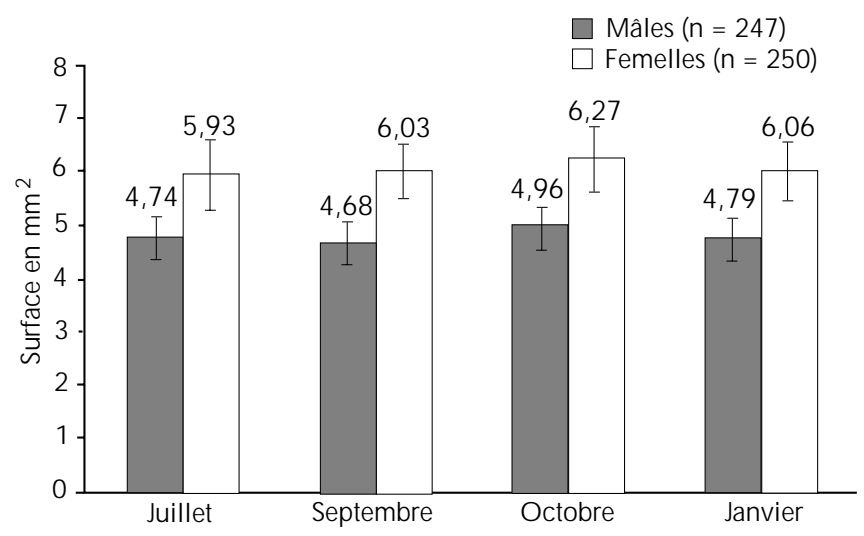

Figure 2 : évolution saisonnière de la surface du thorax chez Glossina palpalis pal palis à Zoukougbeu.

\section{Tableau I}

Probabilité associée au test de Student sur la comparaison des mesures des surfaces du thorax de Glossina palpalis palpalis à Krikoria (Côte d'Ivoire)

\begin{tabular}{lcc} 
Comparaison entre mois & Mâles & Femelles \\
\hline Juillet/septembre & $\mathrm{P}=0,16 \mathrm{~ns}^{*}$ & $\mathrm{P}=0,17 \mathrm{~ns}$ \\
Juillet/octobre & $\mathrm{P}<10-3$ & $\mathrm{P}<0,01$ \\
Juilllet/janvier & $\mathrm{P}=0,27 \mathrm{~ns}$ & $\mathrm{P}=0,15 \mathrm{~ns}$ \\
Septembre/octobre & $\mathrm{P}<10-3$ & $\mathrm{P}<0,05$ \\
Septembre/Janvier & $\mathrm{P}=0,07 \mathrm{~ns}$ & $\mathrm{P}=0,37 \mathrm{~ns}$ \\
Octobre/janvier & $\mathrm{P}<0,05$ & $\mathrm{P}<0,05$
\end{tabular}

* Non significatif

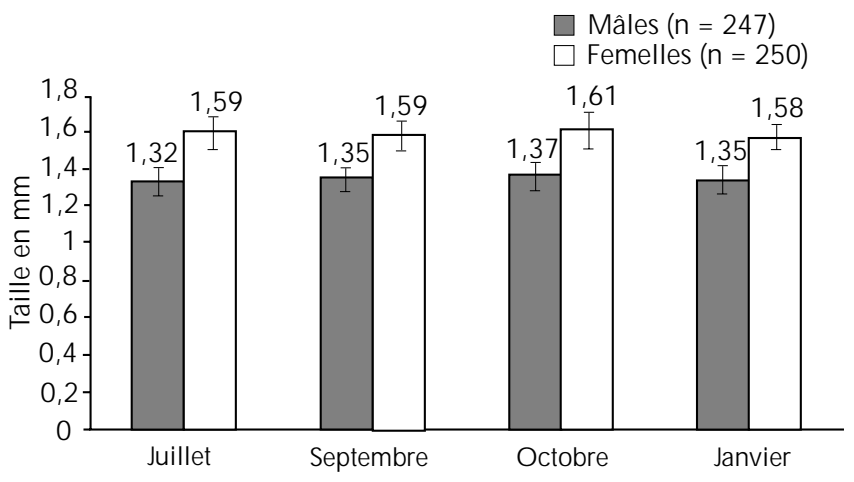

Figure 3 : variations saisonnières de la taille de la cellule en hache chez Glossina palpalis palpalis à Zoukougbeu. 


\section{Tableau II}

Probabilité associée au test de Student sur la comparaison des mesures de la cellule en hache des ailes de Glossina palpalis palpalis à Krikoria (Côte d'Ivoire)

\begin{tabular}{lcc} 
Comparaison entre mois & Mâles & Femelles \\
\hline Juillet/septembre & $\mathrm{P}<0,05$ & $\mathrm{P}=0,44 \mathrm{~ns}$ \\
Juillet/octobre & $\mathrm{P}<10-3$ & $\mathrm{P}=0,07 \mathrm{~ns}$ \\
Juilllet/janvier & $\mathrm{P}<0,05$ & $\mathrm{P}=0,28 \mathrm{~ns}$ \\
Septembre/octobre & $\mathrm{P}=0,06 \mathrm{~ns}$ & $\mathrm{P}=0,08 \mathrm{~ns}$ \\
Septembre/janvier & $\mathrm{P}=0,41 \mathrm{~ns}$ & $\mathrm{P}=0,22 \mathrm{~ns}$ \\
Octobre/janvier & $\mathrm{P}=0,13 \mathrm{~ns}$ & $\mathrm{P}<0,05$
\end{tabular}

* Non significatif

\section{- DISCUSSION ET CONCLUSION}

Les mesures effectuées dans cette étude ont porté sur la taille de la cellule en hache de l'aile et la surface du thorax. Ces paramètres sont proportionnels à la taille de l'insecte (2). La taille des glossines est en relation étroite avec l'humidité observée deux mois auparavant, c'està-dire avec la période du dépôt de la larve (8). D'autres facteurs abiotiques, comme la température, ont un impact important sur la durée de la pupaison des glossines et la taille de l'individu émergent $(4,5$, 16,18 ). En effet, les températures élevées (ou très basses) provoquent une forte déshydratation de la nymphe qui épuise ses réserves lipidiques pour se maintenir en vie et parvient au stade imaginal amoindri physiologiquement et physiquement $(3,8,12)$. Ces variations de taille sont toutefois beaucoup plus limitées que chez d'autres groupes, cet insecte pupipare offrant des conditions de nutrition favorables à sa larve (12).

La taille d'un individu, lors de sa capture, est ainsi directement liée aux conditions écologiques dans lesquelles sa «mère » a vécu (2). Ce paramètre devient ainsi un indicateur de l'état physiologique de la population qui existait environ deux mois auparavant (20).

Des variations de taille ont été mises en évidence dans cette étude, tout d'abord entre les mâles et les femelles. Les valeurs obtenues chez les femelles ont toujours été significativement plus grandes que chez les mâles.

La grande taille de G. p. palpalis observée en saison humide (octobre), également remarquée par Dejardin et Maillot (8) sur Glossina fuscipes quanzensis, pouvait donc être due aux conditions climatiques qui, deux mois auparavant, avaient favorisé un développement optimal des pupes et des populations imaginales. En saison humide, qui commence en août, les conditions climatiques sont homogènes entre les différents biotopes (entre 25 et $27{ }^{\circ} \mathrm{C}$ de température et 70 p. 100 d'humidité) et favorisent la longévité des glossines (14). De la même manière, les tailles minimales observées en saison sèche et en saison chaude (janvier et juillet) traduisent les conditions défavorables que subissent les populations mères. En d'autres termes, les individus plus grands de la saison humide pouvaient refléter une population en meilleur état physiologique que celle vivant en saison sèche (20). La détermination précise de l'âge des glossines étudiées pourrait affiner la connaissance de la période de larviposition des glossines mères. Il n'est en effet pas impossible que dans les populations étudiées aient figuré des glossines d'âges différents, des pupes ayant ainsi été déposées dans le sol à des époques distinctes. Il est intéressant de constater que les mesures de l'aile ou du thorax permettent de différencier des populations au cours du temps, au sein d'une même espèce, voire d'une même sous-espèce, de glossine. La méthodologie utilisée dans ce travail pourrait être améliorée, car des méthodes récentes d'analyse morphométrique, utilisant des logiciels semi-automatisés, ont été mises au point $(1,9)$. Les premiers résultats, prometteurs, laissent également penser qu'il est possible de différencier des populations ayant connu des conditions écologiques distinctes, au sein d'une même espèce, avec des conséquences possibles sur la capacité des glossines à transmettre les trypanosomoses (de la Rocque et coll., résultats non publiés). Avec l'apport récent d'outils moléculaires permettant des études de génétique des populations au niveau intraspécifique (22), la combinaison des informations génétiques et morphométriques permettra l'acquisition d'informations innovantes sur les populations naturelles de glossines, afin de mieux déterminer le risque de transmission des trypanosomoses africaines.

\section{Remerciements}

Ce travail a bénéficié du soutien moral et matériel du Pr Dagnogo Mamadou à l'université de Bouaké (Côte d'Ivoire) que nous remercions vivement.

\section{BIBLIO GRAPHIE}

1. BORNE F., PETITEAU L., GEOFFROY B., DE LA ROCQUE S. CUISANCE D., 1999. Fly Picture Measurement, un nouvel outil informatique pour l'étude des glossines. Revue Elev. Méd. vét Pays trop., $52: 19-21$

2. BURSELL E., 1960. The measurement of size in tsetse flies. Bull. Ent Res., 51: 33-37.

3. BURSELL E., 1960. The effect of temperature on the consumption of fat during pupal development in Glossina. Bull. Ent. Res., 51: 583-598.

4. BUXTON P.A., 1955. The natural history of tsetse flies. London, UK, London School of Hygiene and Tropical Medicine, 816 p. (Memoir N o. 10)

5. CHALLIER A., 1973. Ecologie de Glossina palpalis gambiensis Vanderplank, 1949 (Diptera Muscidae) en savane d'Afrique occidentale. Paris, France, O rstom, 290 p. (coll. M émoires 0 rstom n ${ }^{\circ} 64$ )

6. CHALLIER A., 1973. La capacité vectorielle des glossines. BoboDioulasso, Haute Volta, Centre Muraz, 0 ccge, 22 p. ( n²4/ENT/73)

7. CHALLIER C., EYAUD M., LAFAYE A., LAVEISSIERE C., 1977. Amélioration du rendement du piège biconique pour glossines (Diptera, Glossinidae) par emploi d'un cône inférieur bleu. Cah. O rstom, Sér. Ent. méd. Parasitol., 15 : 283-286.

8. DEJARDIN J., MAILLOT L., 1964. Biométrie de la glossine. Etude statistique des mensurations de l'aile dans diverses communautés (Glossina fuscipes quanzensis, Pires). Revue Elev. Méd. vét Pays trop. 17 : 97-102.

9. DE LA ROCQUE S., GEOFFROY B., CUISANCE D., 1996. Nouvelle approche pour l'estimation de l'âge des glossines par analyse d'image de I'aile. Revue Elev. Méd. vét Pays trop., 49 : 46-48.

10. DRANSFIELD R.D., BRIGHTWELL R., 1989. Problems of field testing theoretical models: a case study. Ann. Soc. belge Méd. trop. 69: 147-154.

11. GLASGOW J.P., 1961. Seasonal variations in size and colour and daily changes in the distribution of Glossina pallidipes Austen in the South Busoga forest U ganda. Bull. ent. Res., 52: 647-666.

12. GLASGOW J.P., 1961. Selection for size in tsetse flies. J. Anim. Ecol., 30: 87-94.

13. GLASGOW J.P., BURSELL E., 1961. Seasonal variations in the fat content and size of Glossina swynnertoni Austen. Bull. ent. Res., 51: 705-713.

14. GOUTEUX J.P., LAVEISSIERE C., 1982. Ecologie des glossines en secteur préforestier de Côte d'Ivoire. 4. Dynamique de l'écodistribution en terroir villageois. Cah. O rstom, Sér. Ent. méd. Parasitol., 20 : 199-229.

15. GOUTEUX J.P., LAVEISSIERE C., BO REHAM P.F.L., 1982. Ecologie des glossines en secteur pré-forestier de Côte d'Ivoire. 2. Les préférences trophiques de Glossina pallicera et $\mathrm{G}$. nigrofusca. Comparaison avec G. palpalis et implications épidémiologiques. Cah. O rstom, Sér. Ent. méd. Parasitol., 20 : 109-124. 
Wing and thorax size of $\mathrm{G}$. palpalis palpalis

16. GRUVEL J., 1974. Contribution à l'étude écologique de Glossina tachinoides Westwood, 1850 (Diptera, Muscidae) dans la réserve de Kalamaloué, vallée du Bas-Chari. Thèse Doct. ès-Sciences naturelles, Université Paris VI, Paris, France, $302 \mathrm{p}$.

17. JACKSON C.H.N., 1953. Seasonal variations in the mean size of tsetse flies. Bull. ent. Res., 43: 703-706.

18. LAVEISSIERE C KIENOU JP. TRAORE T, 1984. Ecologie de Glossina tachinoides Westwood, 1850, en savane humide d'Afrique de I'O uest. X. Durée du stade pupal. Importance de ce paramètre dans la dynamique des populations. Cah. O rstom, Sér. Ent. méd. Parasitol. $22: 219-230$

19. MULLIGAN H.W., 1970. Allen, Undwin Eds., The African trypanosomiases. London, UK, Mulligan, $950 \mathrm{p}$.

\section{Summary}

Sané B., Solano P., Garcia A., Fournet F., Laveissière C. Instraspecific variations of wing and thorax size of Glossina palpalis palpalis (Robineau-Desvoidy, 1830) in the forest region of Côte d'Ivoire

Thorax and wing morphometry was studied in 497 Glossina palpalis palpalis caught on the outskirts of a village in the center-west of Côte d'Ivoire. Significant size differences were observed depending on the sex and season of catching. Tsetse flies caught at the end of the rainy season were bigger than those caught during the dry season. Within a G. p. palpalis population, the relation between climatic conditions and a fly might affect the latter's vectorial capacity. New analytical methods in size measurement will help further specify these results.

Key words: Glossina palpalis palpalis - Wing - Thorax M easurement - Climatic factor - Côte d'Ivoire.
20. PO LLO CK J.N., 1980. Manuel de lutte contre les tsé-tsé. Rome, Italie, Fao, $259 \mathrm{p}$.

21. RO GERS D.J., RAN D O LPH S.E., 1991. Mortality rates and population density of tsetse flies correlated with satellite imagery. Nature, 351: 739-741.

22. SOLANO P., DE LA ROCQUE S., CUISANCE D., GEOFFROY B., DE MEEUS T., CUNY G., DUVALLET G., 1999. Intraspecific variability in natural populations of Glossina palpalis gambiensis from West Africa revealed by genetic and morphometric analyses. Med. vet. Entomol., 13: 401-407.

Reçu le 07.03.2000, accepté le 02.02.2001

\section{Resumen}

Sané B., Solano P., Garcia A., Fournet F., Laveissière C. Variaciones intra específicas del tamaño de las alas y del tórax en Glossina palpalis palpalis (Robineau-Desvoidy, 1830) en la zona forestal de Costa de Marfil

Se estudiaron la morfometría del tórax y de las alas en 497 individuos de Glossina palpalis palpalis, capturados al borde de un pueblo en el centro-oeste de la Costa de Marfil. Se encontraron diferencias significativas en el tamaño, según el sexo y la época de captura. Al final de la estación de las lluvias las glosinas presentaron un tamaño mayor que aquellas capturadas durante la estación seca. La relación entre el tamaño de los individuos que componen una población de glosinas y las condiciones climáticas podría tener consecuencias sobre la capacidad vectorial. Descubrimientos recientes en los métodos de análisis permitirán la mejor definición de estos estudios.

Palabras clave: Glossina palpalis palpalis - Ala - Torax Medición - Factor climatico - Côte d'Ivoire. 